\title{
Nível de ativação e qualidade de vida relacionada à saúde de pessoas em hemodiálise
}

\author{
Level of activation and quality of life related to the health of people in hemodialysis
} Nivel de activación y la calidad de vida relacionada com la salud de personas en hemodiálisis

Denise Rocha Raimundo Leone ${ }^{1}$ (D)

Gabriela Amorim Pereira ${ }^{1}$ (1)

Arison Cristian de Paula Silva² (1)

Aline Silva de Aguiar $^{3}$ (D)

1. Universidade Federal de Juiz de Fora, Faculdade de Medicina Curso de Doutorado em Saúde Coletiva, Programa de PósGraduação Stricto Sensu em Saúde Coletiva. Juiz de Fora, MG, Brasil.

2. Universidade do Estado do Rio de Janeiro, Faculdade de Enfermagem, Residência de Enfermagem em Nefrologia. Rio de Janeiro, RJ, Brasil.

3. Universidade Federal de Juiz de Fora, Departamento de Nutrição do Instituto de Ciências Biológicas. Juiz de Fora, MG, Brasil.

Autor correspondente:

Denise Rocha Raimundo Leone.

E-mail: de_rocha@ymail.com.

Recebido em 10/12/2020.

Aprovado em 09/02/2021.

DOI:https://doi.org/10.1590/2177-9465-EAN-2020-0486

\section{RESUMO}

Objetivo: associar o nível de ativação com a qualidade de vida relacionada à saúde de pessoas que realizam o tratamento hemodialítico. Método: estudo quantitativo, transversal e correlacional com 162 pessoas em tratamento hemodialítico. Os dados foram coletados por meio da aplicação de questionários para a caracterização sociodemográfica, socioeconômica e clínica do Kidney Disease Quality of Life Short Form e da escala Patient Activation Measure. Os dados secundários foram coletados por meio do prontuário médico. Para a análise dos dados, utilizaram-se a estatística descritiva e a regressão logística. Resultados: a ativação do paciente em hemodiálise associou-se positivamente com os domínios sintomas, funcionamento físico, saúde geral, bem-estar emocional, energia/fadiga e o componente mental da qualidade de vida relacionada à saúde. Conclusão e implicação para a prática: como a ativação apresenta relação com a qualidade de vida relacionada à saúde, na prática assistencial, essa métrica deve ser considerada ao implementar medidas que visem a aumentar a qualidade de vida relacionada à saúde das pessoas em hemodiálise.

Palavras-chave: Autogestão; Autocuidado; Participação do Paciente; Hemodiálise; Qualidade de Vida.

\section{Abstract}

Objective: to associate the level of activation with the health-related quality of life of people undergoing hemodialysis. Method: a quantitative, cross-sectional and correlational study with 162 people on hemodialysis treatment. Data was collected by applying questionnaires for sociodemographic, socioeconomic and clinical characterization of the Kidney Disease Quality of Life Short Form and the Patient Activation Measure scale. Secondary data were collected from medical records. For data analysis, descriptive statistics and logistic regression were used. Results: hemodialysis patient activation was positively associated with the domains symptoms, physical functioning, general health, emotional well-being, energy/fatigue and the mental component of health-related quality of life. Conclusion and implication for the practice: since activation is related to health-related quality of life, in care practice, this metric should be considered when implementing measures to increase the health-related quality of life of people on hemodialysis.

Keywords: Self-management; Self-care; Patient Participation; Hemodialysis; Quality of Life

\section{Resumen}

Objetivo: asociar el nivel de activación con la calidad de vida relacionada con la salud de las personas en hemodiálisis. Método: estudio cuantitativo, transversal y correlacional con 162 personas en hemodiálisis. Los datos se recolectaron mediante la aplicación de cuestionarios para la caracterización sociodemográfica, socioeconómica y clínica del Kidney Disease Quality of Life Short Form y la escala Patient Activation Measure. Los datos secundarios se recopilaron a través de historias clínicas. Para el análisis de los datos se utilizó la estadística descriptiva y la regresión logística. Resultados: la activación del paciente en hemodiálisis se asoció positivamente con los dominios síntomas, funcionamiento físico, salud general, bienestar emocional, energía / fatiga y el componente mental de la calidad de vida relacionada con la salud. Conclusión e implicación para la práctica: dado que la activación presenta una relación con la calidad de vida relacionada con la salud, en la práctica asistencial esta métrica debe ser considerada a la hora de implementar medidas dirigidas a incrementar la calidad de vida relacionada con la salud de las personas en hemodiálisis.

Palabras clave: Autogestión; Autocuidado; Participación del paciente; Hemodiálisis; Calidad de vida. 


\section{INTRODUÇÃO}

A hemodiálise (HD) é o tipo de depuração renal mais utilizado globalmente. No Brasil, estimou-se que, em 2018, havia 123.187 pessoas em tratamento hemodialítico, o que corresponde a 92,3\% dos pacientes em diálise crônica. ${ }^{1}$ Ao considerar toda a região da América Latina, a HD é o método de Terapia Renal Substitutiva (TRS) realizado por $75 \%$ dos pacientes com Doença Renal Crônica (DRC) terminal. ${ }^{2}$ Nos Estados Unidos, a HD foi o tratamento de $62,7 \%$ das pessoas com DRC no ano de $2017^{3}$ e, no Reino Unido, mais de 25 mil pessoas realizam esse tratamento. ${ }^{4}$

Embora a HD proporcione a sobrevivência da pessoa com DRC terminal, os pacientes que realizam esse tratamento reportam inúmeras dificuldades, entre elas, o gerenciamento dos sintomas da DRC, as modificações do estilo de vida decorrentes da necessidade de deslocamento ao centro de diálise, as restrições alimentares e hídricas, o comprometimento das atividades laborais, das atividades físicas e do lazer. Esses fatores interferem no cotidiano do paciente em HD e, consequentemente, na sua Qualidade de Vida Relacionada à Saúde (QVRS). . $^{5-7}$

Nesse contexto, conhecer os fatores que se associam à QVRS de pacientes em tratamento hemodialítico é importante, pois, ao se mensurar a QVRS, se define a carga da doença, das lesões e/ou incapacidade para os indivíduos, ${ }^{8}$ além de refletir como se dá o enfrentamento do paciente e quais são as implicações frente à doença e ao seu tratamento. ${ }^{9}$ Além disso, ajuda no direcionamento da atuação do profissional de saúde quanto às variáveis que necessitam ser estimuladas ou alteradas para a obtenção de uma melhor QVRS.

Há evidências de que as pessoas em HD possuem pior QVRS quando comparadas aos pacientes renais crônicos que realizam outro método de TRS para a DRC. ${ }^{10,11} \mathrm{E}$ que uma maior ativação do paciente com doenças crônicas está relacionada à melhor QVRS. ${ }^{12}$

A ativação do paciente refere-se à capacidade e disposição dele em autogerenciar eficazmente a sua saúde e subdivide-se em quatro níveis que consideram o conhecimento, as habilidades e a motivação das pessoas com os cuidados com a sua saúde. ${ }^{13}$ Assim, ativar adultos que possuam doenças crônicas não transmissíveis para a participação do paciente nas atividades de autogestão é essencial para a melhoria da saúde geral e para a QVRS. ${ }^{14}$

Em revisão sistemática que identificou, por meio de 36 artigos, as variáveis que mais influenciavam a QVRS de pessoas em diálise renal, a variável ativação não foi utilizada. ${ }^{15}$ Além disso, estudos que associam a ativação do paciente com a QVRS de pessoas com DRC são limitados ${ }^{16,17} \mathrm{e}$, ao considerar pessoas em tratamento hemodialítico, são ainda mais restritos. ${ }^{17}$ Nesse sentido, pouco se sabe sobre a relação entre a ativação do paciente em HD com a sua QVRS.

$\mathrm{E}$, ao considerar que a ativação se relaciona positivamente com a melhor saúde autorreferida de pacientes em $\mathrm{HD}^{17}$ e com uma menor carga de sintomas de pacientes em todos os estágios da $\mathrm{DRC}$, inclusive daqueles em diálise, ${ }^{4}$ ela pode ser um fator que interfere na QVRS. ${ }^{4,17}$ Nesse sentido, a questão que norteou este estudo foi: "Qual a associação entre o nível de ativação e a QVRS de pessoas em hemodiálise?". Tem-se como hipótese que um maior nível de ativação é favorável a uma melhor QVRS. Assim, objetivou-se associar o nível de ativação com a QVRS de pessoas que realizam o tratamento hemodialítico.

\section{MÉTODO}

Estudo quantitativo, transversal e correlacional realizado em um serviço de hemodiálise localizado em um município da Zona da Mata Mineira, no Estado de Minas Gerais, Brasil. O serviço tem capacidade para atendimento de 240 pacientes e funciona em três turnos, sendo o primeiro de seis às dez horas, o segundo de 11 às 15 horas e o terceiro de 16 às 20 horas.

Foram convidados a participar do estudo todos os 221 pacientes que realizavam HD, entretanto, após aplicar os critérios de elegibilidade, que foram ser maior de 18 anos, lúcido e orientado, que se expressasse por linguagem verbal e que estivesse em HD por um período superior a três meses, conforme relatado por enfermeiros do serviço de HD, e os critérios de exclusão, que foram encontrar-se internado, estar em trânsito, ou seja, realizando temporariamente o tratamento hemodialítico em outro serviço de diálise por conta de viagem, ou ser transplantado durante o período de coleta de dados, constituiu-se a amostra do estudo com 162 pacientes (taxa de resposta: 73,3\%).

Salienta-se que, das 59 pessoas que foram excluídas, 26 não apresentavam capacidade cognitiva para responder às entrevistas, 19 negaram-se a participar, quatro encontravam-se internadas, duas estavam em trânsito, duas transplantaram e seis faleceram.

A coleta de dados ocorreu entre os meses de janeiro e abril de 2019. Os participantes foram recrutados durante o período em que realizavam a HD. Após o aceite em participar da pesquisa e a assinatura do Termo de Consentimento Livre e Esclarecido (TCLE), foi realizada a coleta de dados primários por meio de entrevista estruturada e dos dados secundários por meio de análise dos prontuários.

Como instrumento para a coleta de dados, foi utilizado um questionário estruturado para a avaliação sociodemográfica e clínica, o qual continha questões relacionada à idade, cor da pele, escolaridade, renda, estado civil, ocupação, necessidade de cuidador para a realização das Atividades de Vida Diárias (AVD), hábito tabágico, etilismo, tempo de HD, doença de base, tipo de acesso vascular, realização de outro tipo de TRS, número de comorbidades, presença de diurese renal residual, uso contínuo de medicação, entre outras.

Para a avaliação socioeconômica dos participantes, foi aplicado o questionário de classificação socioeconômica e familiar da Associação Brasileira de Empresas e Pesquisa (APEP), que classifica os domicílios em seis estratos, A, B1, B2, C1, C2, D-E, por meio da mensuração dos itens de conforto que as pessoas possuem em suas residências, da escolaridade do chefe da família, do tipo de pavimento da rua onde se encontra 
a residência do participante e da proveniência da água que abastece o domicílio. ${ }^{18}$

A QVRS foi mensurada pelo Kidney Disease Quality of Life Short Form (KDQOL-SF). Trata-se de um instrumento de avaliação de qualidade de vida específico para pessoas em tratamento dialítico. É composto por 24 questões que englobam 80 itens relacionados à doença renal, aos efeitos da doença renal na qualidade de vida, ao fardo da doença renal, ao status de trabalho, à função cognitiva, à qualidade da interação social, à saúde física e mental e à saúde geral do indivíduo. Para a obtenção do resultado, os dados de cada dimensão são convertidos em escores que variam de zero a 100 , sendo que quanto mais próximo ao 100, melhor é a qualidade de vida. ${ }^{19,20}$

A ativação do paciente foi avaliada por meio do Patient Activation Measure ${ }^{\circledR}$ de 13 itens (PAM-13 ${ }^{\circledR}$ ), adaptado e validado para a utilização no Brasil, ${ }^{21}$ que mensura o conhecimento, a habilidade e a confiança no autogerenciamento do indivíduo. Trata-se de uma escala tipo Likert com 13 questões e cinco opções de respostas. Os pontos obtidos com a aplicação da escala são convertidos no escore de ativação, que se encontra entre zero e 100 pontos, sendo que quanto mais próximo de 100 , mais ativado o paciente está. ${ }^{13,22,23}$

Conforme o escore de ativação, o paciente é classificado em quatro níveis. No nível 1 (pontuação entre 0 - 47,0), são considerados os pacientes que não compreendem a importância do seu papel no autogerenciamento da saúde; no nível 2 (escore de ativação entre 47,1 - 55,1), faltam, no indivíduo, a autoconfiança e o conhecimento para agir; no nível 3 (PAM - 13 entre 55,2 - 72,4), os pacientes envolvem-se nos comportamentos de saúde que são recomendados e, no nível 4 (ativação entre 72,5 - 100), as pessoas são proativas no que diz respeito à sua saúde, além de terem muitos comportamentos favoráveis à saúde. ${ }^{22}$

Os dados secundários, referentes aos valores de hemoglobina e KTV, foram coletados dos prontuários dos participantes e utilizados como fatores de ajuste na análise dos dados.

A análise estatística dos dados deu-se por meio dos softwares Statistical Package for the Social Science SPSS ${ }^{\circledR}$, versão 23.0, e STATA, versão 13.0. Foi adotada a significância estatística de $5 \%$. Para a análise das características sociodemográficas e clínicas, utilizou-se a estatística descritiva, sendo que as variáveis quantitativas foram apresentadas por mediana e intervalo interquartil, visto que elas obtiveram distribuição assimétrica, e as variáveis categóricas, em valores relativos e absolutos.

Para avaliar a relação entre a ativação e a QVRS, foi aplicada a regressão logística. Foram considerados com maior QVRS os participantes com pontuação classificada acima do percentil 50 em cada um dos domínios do KDQOL-SF. Para tanto, estimou-se o Odds Ratio (OD) de maior QV conforme a variável independente, que foi o nível de ativação.

Em um primeiro momento, realizou-se a regressão logística simples para todos os domínios da QVRS e, posteriormente, para os domínios em que a razão de prevalência apresentou significância menor que 0,05, foram feitos ajustes por possíveis fatores de confusão. O primeiro ajuste foi feito para sexo e idade e o segundo, para sexo, idade, tempo de hemodiálise, número de comorbidades, hemoglobina e KTV. Optou-se por utilizar essas variáveis de ajuste, pois há evidências que elas podem estar relacionadas à QVRS. ${ }^{24}$ Para a medida de efeito OD, fol calculado um Intervalo de Confiança de 95\% (IC 95\%).

Foram observados todos os aspectos éticos que envolvem as pesquisas com seres humanos e, somente após a aprovação do Comitê de Ética em Pesquisa de número 3.089.035 e CAAE: 02592418.4.0000.5147, que ocorreu em dezembro de 2018, iniciou-se a coleta de dados.

\section{RESULTADOS}

Dos 162 participantes do estudo, a maioria (62,9\%) era composta por homens, com 60 anos ou mais (53\%), que se autodeclararam negros ou pardos $(61,7 \%)$ e possuíam o Ensino Fundamental incompleto ou eram analfabetos (55\%). Houve o predomínio de pessoas que não possuíam companheiro $(50,6 \%)$, que não residiam sozinhas (83,9\%) e beneficiárias do Instituto Nacional de Segurança Social (INSS) $(85,2 \%)$.

Quanto à renda pessoal, 30,3\% dos entrevistados recebiam três ou mais salários-mínimos. Já em relação à classificação do domicílio, houve a preponderância dos domicílios classificados como C2 (25,3\%). A maior parte dos entrevistados não precisava de acompanhante para o deslocamento ao serviço de HD (76\%) e não necessitava de cuidador para o auxílio e/ou a realização das AVD (88,4\%).

Em relação ao nível de ativação, 23,5\% dos participantes foram classificados como nível 1 de ativação; 29\%, nível 2; 18\%, nível 3 e 29\%, nível 4 . A caracterização sociodemográfica dos entrevistados, conforme o nível de ativação, encontra-se descrita na Tabela 1.

No que se refere às variáveis clínicas, predominou, como doença de base para a DRC, a hipertensão arterial, que foi diagnosticada em $40,3 \%$ dos participantes. Além disso, 50,6\% apresentaram uma comorbidade, $72,7 \%$ faziam $\mathrm{HD}$ por um período inferior a cinco anos, 61,9\% utilizavam a fístula arteriovenosa como acesso vascular, $92 \%$ não haviam realizado outro tipo de Terapia Renal Substitutiva previamente, 75,3\% apresentavam diurese renal residual, 91,4\% faziam uso de medicação contínua, $90,1 \%$ relataram não ser tabagista e $84,6 \%$ informaram não ser etilistas.

Em relação aos exames laboratoriais, com exceção da hemoglobina, cujos $61,1 \%$ dos pacientes em HD apresentaram níveis séricos inadequados, em todos os outros eletrólitos, houve o predomínio dos níveis séricos adequados, sendo 50,5\% para o potássio e fósforo, $75,9 \%$ para o cálcio e $79,6 \%$ o sódio. 0 KTV ideal $(<1,2)$ esteve presente em $72,2 \%$ dos participantes. Além disso, na maior parte desses $(85,8 \%)$, o ganho do intervalo interdialítico foi igual ou inferir a $5 \%$ do peso seco. A categorização dos dados clínicos, de acordo com o nível de ativação dos pacientes, encontra-se na Tabela 2.

No que se refere à QVRS, na Tabela 3, encontram-se os valores da média, mediana, intervalo interquartil e valores mínimo e máximo de todos os domínios da QVRS que são 
Tabela 1. Caracterização sociodemográfica dos pacientes em hemodiálise ( $n=162)$ de acordo com o nível de ativação, Juiz de Fora, MG, Brasil, 2019.

\begin{tabular}{|c|c|c|c|c|c|c|c|c|}
\hline \multirow{3}{*}{ Dados Sociodemográficos } & \multicolumn{8}{|c|}{ Nível PAM } \\
\hline & \multicolumn{2}{|c|}{ Nível 1} & \multicolumn{2}{|c|}{ Nível 2} & \multicolumn{2}{|c|}{ Nível 3} & \multicolumn{2}{|c|}{ Nível 4} \\
\hline & $\mathrm{n}$ & $\%$ & $n$ & $\%$ & $n$ & $\%$ & $n$ & $\%$ \\
\hline \multicolumn{9}{|l|}{ Gênero } \\
\hline Masculino & 24 & $14,8 \%$ & 29 & $17,9 \%$ & 20 & $12,3 \%$ & 29 & $17,9 \%$ \\
\hline Feminino & 14 & $8,7 \%$ & 18 & $11,1 \%$ & 10 & $6,2 \%$ & 18 & $11,1 \%$ \\
\hline \multicolumn{9}{|l|}{ Idade } \\
\hline Abaixo de 60 anos & 21 & $13 \%$ & 16 & $9,9 \%$ & 17 & $10,5 \%$ & 22 & $13,6 \%$ \\
\hline 60 anos ou mais & 17 & $10,5 \%$ & 31 & $19,1 \%$ & 13 & $8 \%$ & 25 & $15,4 \%$ \\
\hline \multicolumn{9}{|l|}{ Cor da pele } \\
\hline Não Branca (negra e parda) & 29 & $17,9 \%$ & 29 & $17,9 \%$ & 19 & $11,7 \%$ & 23 & $14,2 \%$ \\
\hline Branca & 9 & $5,6 \%$ & 18 & $11,1 \%$ & 11 & $6,8 \%$ & 24 & $14,8 \%$ \\
\hline \multicolumn{9}{|l|}{ Anos de estudo } \\
\hline Analfabeto ou fundamental incompleto & 25 & $15,4 \%$ & 30 & $18,6 \%$ & 13 & $8 \%$ & 21 & $13 \%$ \\
\hline Ensino Fundamental completo & 2 & $1,2 \%$ & 3 & $1,8 \%$ & 3 & $1,8 \%$ & 5 & $3,1 \%$ \\
\hline Ensino Médio ou Técnico & 9 & $5,6 \%$ & 12 & $7,4 \%$ & 10 & $6,2 \%$ & 10 & $6,2 \%$ \\
\hline Ensino Superior & 2 & $1,2 \%$ & 2 & $1,2 \%$ & 4 & $2,5 \%$ & 11 & $6,8 \%$ \\
\hline \multicolumn{9}{|l|}{ Situação conjugal } \\
\hline Com companheiro & 17 & $10,5 \%$ & 21 & $13 \%$ & 16 & $9,9 \%$ & 26 & $16 \%$ \\
\hline Sem companheiro & 21 & $13 \%$ & 26 & $16 \%$ & 14 & $8,6 \%$ & 21 & $13 \%$ \\
\hline \multicolumn{9}{|l|}{ Reside Sozinho } \\
\hline Sim & 4 & $2,5 \%$ & 9 & $5,6 \%$ & 5 & $3,1 \%$ & 8 & $4,9 \%$ \\
\hline Não & 34 & $21 \%$ & 38 & $23,4 \%$ & 25 & $15,4 \%$ & 39 & $24,1 \%$ \\
\hline \multicolumn{9}{|l|}{ Beneficiário do INSS } \\
\hline Sim & 35 & $21,6 \%$ & 42 & $26 \%$ & 23 & $14,2 \%$ & 38 & $23,4 \%$ \\
\hline Não & 3 & $1,8 \%$ & 5 & $3,1 \%$ & 7 & $4,3 \%$ & 9 & $5,6 \%$ \\
\hline \multicolumn{9}{|l|}{ Renda Pessoal } \\
\hline 1 salário-mínimo & 7 & $4,3 \%$ & 13 & $8 \%$ & 4 & $2,5 \%$ & 13 & $8 \%$ \\
\hline 2 salários-mínimos & 14 & $8,7 \%$ & 11 & $6,8 \%$ & 6 & $3,7 \%$ & 11 & $6,8 \%$ \\
\hline 3 salários-mínimos & 5 & $3,1 \%$ & 10 & $6,2 \%$ & 7 & $4,3 \%$ & 6 & $3,7 \%$ \\
\hline Acima de 3 salários-mínimos & 10 & $6,2 \%$ & 12 & $7,4 \%$ & 12 & $7,4 \%$ & 15 & $9,3 \%$ \\
\hline Não sabe/Não respondeu & 2 & $1,2 \%$ & 1 & $0,6 \%$ & 1 & $0,6 \%$ & 2 & $1,2 \%$ \\
\hline \multicolumn{9}{|l|}{ Classificação econômica do domicílio } \\
\hline A & 2 & $1,2 \%$ & 1 & $0,6 \%$ & 5 & $3,1 \%$ & 6 & $3,7 \%$ \\
\hline B1 & 1 & $0,6 \%$ & 4 & $2,5 \%$ & 5 & $3,1 \%$ & 9 & $5,6 \%$ \\
\hline B2 & 12 & $7,4 \%$ & 8 & $4,9 \%$ & 3 & $1,8 \%$ & 13 & $8 \%$ \\
\hline $\mathrm{C} 1$ & 9 & $5,6 \%$ & 9 & $5,6 \%$ & 6 & $3,7 \%$ & 9 & $5,6 \%$ \\
\hline $\mathrm{C} 2$ & 8 & $4,9 \%$ & 16 & $9,9 \%$ & 10 & $6,2 \%$ & 7 & $4,3 \%$ \\
\hline D-E & 6 & $3,7 \%$ & 9 & $5,6 \%$ & 1 & $0,6 \%$ & 3 & $1,8 \%$ \\
\hline
\end{tabular}

Fonte: dados de pesquisa. 
Tabela 1. Continued...

\begin{tabular}{|c|c|c|c|c|c|c|c|c|}
\hline \multirow{3}{*}{ Dados Sociodemográficos } & \multicolumn{8}{|c|}{ Nível PAM } \\
\hline & \multicolumn{2}{|c|}{ Nível 1} & \multicolumn{2}{|c|}{ Nível 2} & \multicolumn{2}{|c|}{ Nível 3} & \multicolumn{2}{|c|}{ Nível 4} \\
\hline & $\mathbf{n}$ & $\%$ & $\mathbf{n}$ & $\%$ & $\mathbf{n}$ & $\%$ & $\mathbf{n}$ & $\%$ \\
\hline \multicolumn{9}{|l|}{ Acompanhante para a hemodiálise } \\
\hline Sim & 15 & $9,3 \%$ & 18 & $11,1 \%$ & 3 & $1,8 \%$ & 3 & $1,8 \%$ \\
\hline Não & 23 & $14,2 \%$ & 29 & $17,9 \%$ & 27 & $16,7 \%$ & 44 & $27,2 \%$ \\
\hline \multicolumn{9}{|c|}{$\begin{array}{l}\text { Necessita de cuidador para atividades da vida } \\
\text { diária }\end{array}$} \\
\hline Não & 30 & $18,6 \%$ & 39 & $24,1 \%$ & 28 & $17,3 \%$ & 46 & $28,4 \%$ \\
\hline Sim & 8 & $4,9 \%$ & 8 & $4,9 * \%$ & 2 & $1,2 \%$ & 1 & $0,6 \%$ \\
\hline
\end{tabular}

Fonte: dados de pesquisa.

Tabela 2. Caracterização clínica conforme o nível de ativação dos pacientes em hemodiálise (n=162), Juiz de Fora, MG, Brasil, 2019.

\begin{tabular}{|c|c|c|c|c|c|c|c|c|}
\hline \multirow{2}{*}{ Dados Clínicos } & \multicolumn{2}{|c|}{ Nível 1} & \multicolumn{2}{|c|}{ Nível 2} & \multicolumn{2}{|c|}{ Nível 3} & \multicolumn{2}{|c|}{ Nível 4} \\
\hline & $\mathrm{n}$ & $\%$ & $\mathbf{n}$ & $\%$ & $\mathbf{n}$ & $\%$ & $\mathbf{n}$ & $\%$ \\
\hline \multicolumn{9}{|l|}{ Doença de base } \\
\hline Hipertensão arterial & 15 & $9,3 \%$ & 21 & $13 \%$ & 14 & $8,7 \%$ & 15 & $9,3 \%$ \\
\hline Diabetes & 8 & $4,9 \%$ & 13 & $8 \%$ & 3 & $1,8 \%$ & 4 & $2,5 \%$ \\
\hline Hipertensão arterial + Diabetes Mellitus & 9 & $5,6 \%$ & 7 & $4,3 \%$ & 6 & $3,7 \%$ & 15 & $9,3 \%$ \\
\hline Glomerulopatias & 3 & $1,8 \%$ & 2 & $1,2 \%$ & 1 & $0,6 \%$ & 3 & $1,8 \%$ \\
\hline Outros & 1 & $0,6 \%$ & 0 & $0 \%$ & 3 & $1,8 \%$ & 3 & $1,8 \%$ \\
\hline Não sabe/Não respondeu & 2 & $1,2 \%$ & 4 & $2,5 \%$ & 3 & $1,8 \%$ & 7 & $4,3 \%$ \\
\hline \multicolumn{9}{|l|}{ Número de comorbidades } \\
\hline 1 & 18 & $11,1 \%$ & 25 & $15,4 \%$ & 16 & $9,9 \%$ & 23 & $14,2 \%$ \\
\hline 2 & 16 & $9,9 \%$ & 12 & $7,4 \%$ & 10 & $6,2 \%$ & 22 & $13,5 \%$ \\
\hline$>3$ & 4 & $2,5 \%$ & 10 & $6,2 \%$ & 4 & $2,5 \%$ & 2 & $1,2 \%$ \\
\hline \multicolumn{9}{|l|}{ Tempo de HD } \\
\hline Menor que 5 anos & 28 & $17,2 \%$ & 37 & $22,8 \%$ & 16 & $9,9 \%$ & 37 & $22,8 \%$ \\
\hline 5 anos ou mais & 10 & $6,2 \%$ & 10 & $6,2 \%$ & 14 & $8,7 \%$ & 10 & $6,2 \%$ \\
\hline \multicolumn{9}{|l|}{ Tipo de acesso vascular } \\
\hline Cateter duplo lúmen & 13 & $8 \%$ & 15 & $9,2 \%$ & 14 & $8,7 \%$ & 15 & $9,2 \%$ \\
\hline Fístula arteriovenosa & 24 & $14,8 \%$ & 30 & $18,6 \%$ & 16 & $9,9 \%$ & 30 & $18,6 \%$ \\
\hline Ambos & 1 & $0,6 \%$ & 2 & $1,2 \%$ & 0 & $0 \%$ & 2 & $1,2 \%$ \\
\hline \multicolumn{9}{|l|}{ Terapia Renal Substitutiva prévia } \\
\hline Não & 37 & $22,8 \%$ & 45 & $27,8 \%$ & 26 & $16 \%$ & 41 & $25,4 \%$ \\
\hline Sim & 1 & $0,6 \%$ & 2 & $1,2 \%$ & 4 & $2,5 \%$ & 6 & $3,7 \%$ \\
\hline \multicolumn{9}{|l|}{ Diurese renal residual } \\
\hline Sim & 27 & $16,7 \%$ & 40 & $24,7 \%$ & 17 & $10,5 \%$ & 38 & $23,4 \%$ \\
\hline Não & 11 & $6,8 \%$ & 7 & $4,3 \%$ & 13 & $8 \%$ & 9 & $5,6 \%$ \\
\hline Uso de medicação contínua & & & & & & & & \\
\hline
\end{tabular}

Fonte: dados de pesquisa. 
Tabela 2. Continued...

\begin{tabular}{|c|c|c|c|c|c|c|c|c|}
\hline \multirow{2}{*}{ Dados Clínicos } & \multicolumn{2}{|c|}{ Nível 1} & \multicolumn{2}{|c|}{ Nível 2} & \multicolumn{2}{|c|}{ Nível 3} & \multicolumn{2}{|c|}{ Nível 4} \\
\hline & $\mathbf{n}$ & $\%$ & n & $\%$ & $\mathbf{n}$ & $\%$ & $\mathbf{n}$ & $\%$ \\
\hline Sim & 32 & $19,8 \%$ & 44 & $27,2 \%$ & 29 & $17,9 \%$ & 43 & $26,5 \%$ \\
\hline Não & 4 & $2,5 \%$ & 3 & $1,8 \%$ & 1 & $0,6 \%$ & 4 & $2,5 \%$ \\
\hline Não sabe/não responde & 2 & $1,2 \%$ & 0 & $0 \%$ & 0 & $0 \%$ & 0 & $0 \%$ \\
\hline \multicolumn{9}{|l|}{ Tabagista } \\
\hline Não & 36 & $22,2 \%$ & 42 & $26 \%$ & 25 & $15,4 \%$ & 43 & $26,5 \%$ \\
\hline Sim & 2 & $1,2 \%$ & 5 & $3,1 \%$ & 5 & $3,1 \%$ & 4 & $2,5 \%$ \\
\hline \multicolumn{9}{|l|}{ Etilista } \\
\hline Não & 32 & $19,8 \%$ & 42 & $26 \%$ & 23 & $14,2 \%$ & 40 & $24,6 \%$ \\
\hline Sim & 6 & $3,7 \%$ & 5 & $3,1 \%$ & 7 & $4,3 \%$ & 7 & $4,3 \%$ \\
\hline \multicolumn{9}{|l|}{ KTV } \\
\hline$>1,2$ & 27 & $16,6 \%$ & 42 & $26 \%$ & 21 & $13 \%$ & 27 & $16,6 \%$ \\
\hline$<1,2$ & 11 & $6,8 \%$ & 5 & $3,1 \%$ & 9 & $5,6 \%$ & 20 & $12,3 \%$ \\
\hline \multicolumn{9}{|l|}{ Hemoglobina } \\
\hline$>11 \mathrm{~d} / \mathrm{dL}$ & 11 & $6,8 \%$ & 19 & $11,7 \%$ & 14 & $8,7 \%$ & 19 & $11,7 \%$ \\
\hline$<11 \mathrm{~d} / \mathrm{dL}$ & 27 & $16,6 \%$ & 28 & $17,3 \%$ & 16 & $9,9 \%$ & 28 & $17,3 \%$ \\
\hline \multicolumn{9}{|l|}{ Potássio } \\
\hline Adequado (entre 3,5 e 5,0 mEq/L) & 22 & $13,5 \%$ & 24 & $14,8 \%$ & 19 & $11,7 \%$ & 17 & $10,5 \%$ \\
\hline Inadequado & 16 & $9,9 \%$ & 23 & $14,2 \%$ & 11 & $6,8 \%$ & 30 & $18,6 \%$ \\
\hline \multicolumn{9}{|l|}{ Fósforo } \\
\hline Adequado (entre 3,5 e $5,5 \mathrm{mg} / \mathrm{dL}$ ) & 19 & $11,7 \%$ & 29 & $17,9 \%$ & 12 & $7,4 \%$ & 22 & $13,5 \%$ \\
\hline Inadequado & 19 & $11,7 \%$ & 18 & $11,2 \%$ & 18 & $11,2 \%$ & 25 & $15,4 \%$ \\
\hline \multicolumn{9}{|l|}{ Sódio } \\
\hline Adequado (entre 135 e 145 mmol/L) & 33 & $20,4 \%$ & 33 & $20,4 \%$ & 24 & $14,8 \%$ & 39 & $24 \%$ \\
\hline Inadequado & 5 & $3,1 \%$ & 14 & $8,7 \%$ & 6 & $3,7 \%$ & 8 & $4,9 \%$ \\
\hline \multicolumn{9}{|l|}{ Cálcio } \\
\hline Adequado (entre 8,4 e 10,2 mgdL) & 25 & $15,4 \%$ & 38 & $23,5 \%$ & 23 & $14,2 \%$ & 37 & $22,8 \%$ \\
\hline Inadequado & 13 & $8 \%$ & 9 & $5,6 \%$ & 7 & $4,3 \%$ & 10 & $6,2 \%$ \\
\hline \multicolumn{9}{|c|}{ Ganho de peso no intervalo interdialítico } \\
\hline$<5 \%$ & 33 & $20,4 \%$ & 40 & $24,7 \%$ & 26 & $16 \%$ & 40 & $24,7 \%$ \\
\hline$>5 \%$ & 5 & $3,1 \%$ & 7 & $4,3 \%$ & 4 & $2,5 \%$ & 7 & $4,3 \%$ \\
\hline
\end{tabular}

Fonte: dados de pesquisa.

analisados pelo KDQOL-SF. Salienta-se que os domínios do KDQOL-SF obtiveram distribuição assimétrica, contudo, visando à possibilidade de comparações, optou-se por apresentar também o valor da média.

Ao considerar as dimensões específicas, as piores pontuações foram atribuídas ao status de trabalho e à carga da DRC e as melhores pontuações, ao incentivo do pessoal da diálise e ao suporte social. Observa-se, tendo em conta os domínios das dimensões genéricas, que o componente de saúde física e a função social foram os que pior e melhor pontuaram, respectivamente.

$\mathrm{Na}$ análise univariada, conforme consta na Tabela 4, os pacientes com nível 4 de ativação apresentaram 3,72 vezes mais chances de terem melhor funcionamento físico e melhor saúde geral quando comparados aos participantes com nível 1 de ativação. Além disso, possuíam 2,76 vezes mais chances de ter melhor bem-estar emocional e maior pontuação no domínio sintoma, 4,62 vezes chances de ter mais energia e 3,82 vezes 
Tabela 3. Dimensões da qualidade de vida relacionada à saúde de pacientes em hemodiálise, Juiz de Fora, MG, Brasil, 2019.

\begin{tabular}{|c|c|c|c|c|c|c|}
\hline \multicolumn{7}{|c|}{ Qualidade de vida } \\
\hline Dimensões & Média & Mediana & $\begin{array}{c}\text { Intervalo } \\
\text { Interquartil }\end{array}$ & Mínimo & Máximo & $\mathbf{N}$ \\
\hline \multicolumn{7}{|l|}{ Dimensões Específicas } \\
\hline Sintomas & 77,70 & 81,25 & 20,83 & 31,25 & 100,00 & 162 \\
\hline Efeitos da Doença Renal Crônica & 71,74 & 75,00 & 28,13 & 0,00 & 100,00 & 162 \\
\hline Carga da Doença Renal Crônica & 54,90 & 56,25 & 56,25 & 0,00 & 100,00 & 162 \\
\hline Status de trabalho & 25,00 & 0,00 & 50,00 & 0,00 & 100,00 & 162 \\
\hline Função cognitiva & 81,28 & 86,67 & 26,67 & 13,33 & 100,00 & 162 \\
\hline Interação social & 81,98 & 86,67 & 33,33 & 20,00 & 100,00 & 162 \\
\hline Função sexual & 84,76 & 100,00 & 25,00 & 0,00 & 100,00 & 73 \\
\hline Sono & 69,95 & 72,50 & 38,13 & 5,00 & 100,00 & 162 \\
\hline Suporte social & 86,63 & 100,00 & 16,67 & 0,00 & 100,00 & 162 \\
\hline Incentivo do pessoal da diálise & 86,81 & 100,00 & 25,00 & 0,00 & 100,00 & 162 \\
\hline Satisfação do paciente & 74,07 & 83,33 & 16,67 & 33,33 & 100,00 & 162 \\
\hline \multicolumn{7}{|l|}{ Dimensões Genéricas - SF36 } \\
\hline Saúde global & 68,95 & 70,00 & 30,00 & 0,00 & 100,00 & 162 \\
\hline Função física & 53,46 & 55,00 & 55,00 & 0,00 & 100,00 & 162 \\
\hline Limitação das funções físicas & 42,44 & 50,00 & 75,00 & 0,00 & 100,00 & 162 \\
\hline Dor & 65,12 & 70,00 & 65,00 & 0,00 & 100,00 & 162 \\
\hline Saúde geral & 58,55 & 60,00 & 35,00 & 5,00 & 100,00 & 162 \\
\hline Bem-estar emocional & 69,53 & 72,00 & 37,00 & 4,00 & 100,00 & 162 \\
\hline Papel emocional & 54,53 & 66,67 & 66,67 & 0,00 & 100,00 & 162 \\
\hline Função social & 69,91 & 75,00 & 50,00 & 0,00 & 100,00 & 162 \\
\hline Fadiga/Energia & 58,12 & 62,50 & 41,25 & 0,00 & 100,00 & 162 \\
\hline Componente de saúde física & 40,15 & 42,37 & 16,73 & 14,22 & 58,72 & 162 \\
\hline Componente de saúde mental & 46,31 & 47,90 & 16,47 & 17,33 & 68,68 & 162 \\
\hline
\end{tabular}

Fonte: dados de pesquisa.

mais chances de melhor pontuação no componente mental quando comparados aos pacientes classificados como nível 1 de ativação. No que se refere ao domínio incentivo do pessoal da diálise, aqueles considerados nível 2 de ativação tinham 4,22 vezes chances de ter maior pontuação nesse domínio quando comparados aos pacientes do nível 1.

Após o ajuste para as possíveis variáveis de confundimento, as pessoas altamente ativadas (nível 4) mantiveram maiores chances de possuir melhor QVRS nos domínios sintomas $(\mathrm{OR}=3,11$; IC: $1,21-7,94)$, funcionamento físico $(\mathrm{OR}=4,18$; IC: 1,64-12,24), saúde geral (OR =3,49; IC: 1,39-8,75), bem-estar emocional ( $O R=3,12$, IC: $1,22-8,02)$, energia/fadiga $(O R=4,79$; IC: 1,82-12,55) e componente mental (OR:4,33; IC: 1,68-11,11) em comparação àquelas com o menor nível de ativação (nível 1), conforme a Tabela 5.

\section{DISCUSSÃO}

Neste estudo, pessoas com o nível 4 de ativação tinham mais chances de obter melhor QVRS nos domínios sintomas, funcionamento físico, saúde geral, bem-estar emocional, energia/ fadiga e componente mental quando comparadas àquelas com nível 1 de ativação.

Ao corroborar com os achados deste estudo, a ativação do paciente foi estreitamente relacionada à QVRS em uma coorte inglesa com 3325 pacientes em todos os estágios da DRC. Na referida coorte, utilizou-se o instrumento 5-level EQ-5D Version (EQ5D-5L QoL domains) para mensurar a qualidade de vida. Evidenciou-se que os pacientes com baixa ativação (níveis 1 e 2) apresentavam maiores chances de relatar problemas moderados em todos os domínios que compõem o instrumento, que são mobilidade, autocuidado, realização das atividades usuais, 
Tabela 4. Razão de chances de componentes (OR) e Intervalo de Confiança (IC) de componentes da qualidade de vida relacionada à saúde acima do percentil 50† de acordo com o nível de ativação dos pacientes em HD, Juiz de Fora, MG, Brasil, 2019.

\begin{tabular}{|c|c|c|c|c|}
\hline \multirow{2}{*}{ OR (IC 95\%) } & \multicolumn{4}{|c|}{ Nível de ativação } \\
\hline & 1 & 2 & 3 & 4 \\
\hline Sintoma & 1.0 & $2,12(0,88-5,09)$ & $1,71(0,64-, 53)$ & $2,76(1,14-6,68)^{*}$ \\
\hline Efeito da DRC & 1.0 & $0,69(0,29-1,64)$ & $0,55(0,21-1,46)$ & $0,90(0,37-2,13)$ \\
\hline Carga da DRC & 1.0 & $1,06(0,45-2,50)$ & $0,47(0,17-1,30)$ & $2,37(0,97-5,73)$ \\
\hline Status Trabalho & 1.0 & $1,19(0,48-2,91)$ & $1,47(0,54-3,93)$ & $2,00(0,83-4,84)$ \\
\hline Função Cognitiva & 1.0 & $1,31(0,55-3,11)$ & $1,05(0,39-2,76)$ & $1,07(0,71-4,03)$ \\
\hline Interação Social & 1.0 & $0,61(0,25-1,44)$ & $0,60(0,22-1,58)$ & $1,21(0,51-2,87)$ \\
\hline Função Sexual & 1.0 & $0,54(0,8-3,36)$ & $0,50(0,07-3,54)$ & $0,57(0,09-3,40)$ \\
\hline Sono & 1.0 & $1,94(0,81-4,66)$ & $1,71(0,64-4,53)$ & $1,71(0,64-4,53)$ \\
\hline Suporte Social & 1.0 & $0,93(0,38-2,27)$ & $1,00(0,37-2,71)$ & $2,15(0,82-5,63)$ \\
\hline Incentivo à diálise & 1.0 & $4,22(1,60-11,0) *$ & $0,66(0,25-1,75)$ & $2,1(0,88-5,16)$ \\
\hline Saúde Global & 1.0 & $1,11(0,46-2,63)$ & $0,68(0,25-1,86)$ & $1,11(0,46-2,63)$ \\
\hline Satisfação do Paciente & 1.0 & $0,88(0,37-2,07)$ & $1,50(0,56-3,95)$ & $1,35(0,57-3,18)$ \\
\hline Funcionamento Físico & 1.0 & $1,19(0,48-2,91)$ & $2,51(0,93-6,73)$ & $3,72(1,51-9,18)^{*}$ \\
\hline Função Física & 1.0 & $0,71(0,30-1,68)$ & $0,80(0,31-2,11)$ & $1,09(0,46-2,58)$ \\
\hline Dor & 1.0 & $1,43(0,60-3,39)$ & $0,91(0,34-2,42)$ & $1,85(0,78-4,41)$ \\
\hline Saúde Geral & 1.0 & $2,18(0,90-5,27)$ & $0,96(0,34-2,64)$ & $3,72(1,51-9,18)^{*}$ \\
\hline Bem-estar Emocional & 1.0 & $1,26(0,52-3,05)$ & $1,95(0,73-5,18)$ & $2,76(1,14-6,68)^{*}$ \\
\hline Papel Emocional & 1.0 & $1,04(0,44-2,45)$ & $1,00(0,38-2,60)$ & $1,76(0,73-4,21)$ \\
\hline Função Social & 1.0 & $0,91(0,38-2,17)$ & $0,80(0,31-2,11)$ & $1,90(0,78-4,66)$ \\
\hline Energia/Fadiga & 1.0 & $2,07(0,85-5,06)$ & $1,89(0,70-5,10)$ & $4,62(1,84-11,58)^{*}$ \\
\hline Componente Físico & 1.0 & $1,11(0,46-2,63)$ & $1,79(0,68-4,73)$ & $1,85(0,78-4,41)$ \\
\hline Componente Mental & 1.0 & $2,46(1,006,00)^{*}$ & $1,89(0,70-5,10)$ & $3,82(1,54-9,46)^{*}$ \\
\hline
\end{tabular}

+ Representação da amostra de acordo com os componentes de qualidade de vida com pontuação acima do percentil 500. * p valor $<0,05$. Fonte: dados de pesquisa.

Tabela 5. Razão de chances de componentes da QVRS acima do percentil $50^{+}$de acordo com o nível de ativação dos participantes ajustado por fatores com confusão, Juiz de Fora, MG, Brasil, 2019.

\begin{tabular}{|c|c|c|c|c|}
\hline & \multicolumn{2}{|c|}{ Ajuste $1^{*}$} & \multicolumn{2}{|c|}{ Ajuste $2^{* *}$} \\
\hline & OR (IC 95\%) & Valor $p$ & OR (IC 95\%) & Valor $\mathrm{p}$ \\
\hline & \multicolumn{4}{|c|}{ Sintoma } \\
\hline \multicolumn{5}{|c|}{ Nível de Ativação } \\
\hline 1 & 1.0 & & 1.0 & \\
\hline 2 & $2,19(0,89-5,40)$ & 0,086 & $2,26(0,89-5,73)$ & 0,085 \\
\hline 3 & $1,70(0,63-4,61)$ & 0,293 & $2,05(0,73-5,75)$ & 0,169 \\
\hline 4 & $2,99(1,20-7,41)$ & 0,018 & $3,11(1,21-7,94)$ & 0,018 \\
\hline
\end{tabular}

† Representação da amostra de acordo com os componentes de qualidade de vida com pontuação acima do percentil 50. Domínio Sintoma $\geq 81,25$, n=84; Funcionamento físico $\geq 60, n=79$; Saúde geral $\geq 65, n=79$; Bem-estar emocional $\geq 75, n=79$; Energia/Fadiga $\geq 65, n=81 ;$ Componente mental $\geq 47,95, n=81$.

* Ajuste 1: Sexo e Idade. ** Ajuste 2: Sexo; Idade; Tempo de Hemodiálise; Número de Comorbidades; Hemoglobina e KTV. Fonte: dados de pesquisa. 
Tabela 5. Continued...

\begin{tabular}{|c|c|c|c|c|}
\hline & \multicolumn{2}{|c|}{ Ajuste 1* } & \multicolumn{2}{|c|}{ Ajuste $2 * *$} \\
\hline & OR (IC 95\%) & Valor $p$ & OR (IC 95\%) & Valor $p$ \\
\hline & \multicolumn{4}{|c|}{ Funcionamento Físico } \\
\hline \multicolumn{5}{|c|}{ Nível de Ativação } \\
\hline 1 & 1.0 & & 1.0 & \\
\hline 2 & $1,28(0,50-3,22)$ & 0,597 & $1,03(0,38-2,75)$ & 0,949 \\
\hline 3 & $2,58(0,93-7,14)$ & 0,068 & $3,07(1,02-9,21)$ & 0,045 \\
\hline \multirow[t]{2}{*}{4} & $3,94(1,54-10,08)$ & 0,004 & $4,48(1,64-12,24)$ & 0,003 \\
\hline & \multicolumn{4}{|c|}{ Saúde Geral } \\
\hline \multicolumn{5}{|c|}{ Nível de Ativação } \\
\hline 1 & 1.0 & & 1.0 & \\
\hline 2 & $2,15(0,90-5,25)$ & 0,084 & $2,26(0,91-5,61)$ & 0,079 \\
\hline 3 & $0,96(0,35-2,60)$ & 0,946 & $0,88(0,31-2,50)$ & 0,824 \\
\hline \multirow[t]{2}{*}{4} & $3,75(1,52-9,26)$ & 0,004 & $3,49(1,39-8,75)$ & 0,008 \\
\hline & \multicolumn{4}{|c|}{ Bem-estar emocional } \\
\hline \multicolumn{5}{|c|}{ Nível de Ativação } \\
\hline 1 & 1.0 & & 1.0 & \\
\hline 2 & $1,30(0,52-3,25)$ & 0,572 & $1,24(0,48-3,17)$ & 0,643 \\
\hline 3 & $1,98(0,71-5,49)$ & 0,186 & $1,98(0,70-5,55)$ & 0,191 \\
\hline \multirow[t]{2}{*}{4} & $3,13(1,23-7,94)$ & 0,016 & $3,12(1,22-8,02)$ & 0,018 \\
\hline & \multicolumn{4}{|c|}{ Energia/Fadiga } \\
\hline \multicolumn{5}{|c|}{ Nível de Ativação } \\
\hline 1 & 1.0 & & 1.0 & \\
\hline 2 & $2,20(0,88-5,48)$ & 0,088 & $2,02(0,79-5,14)$ & 0,140 \\
\hline 3 & $1,89(0,69-5,17)$ & 0,215 & $1,77(0,62-5,01)$ & 0,280 \\
\hline \multirow[t]{2}{*}{4} & $4,74(1,85-12,10)$ & 0,001 & $4,79(1,82-12,55)$ & 0,001 \\
\hline & \multicolumn{4}{|c|}{ Componente Mental } \\
\hline \multicolumn{5}{|c|}{ Nível de Ativação } \\
\hline 1 & 1.0 & & 1.0 & \\
\hline 2 & $2,53(1,02-6,23)$ & 0,043 & $2,31(0,92-5,80)$ & 0,074 \\
\hline 3 & $1,87(0,69-5,09)$ & 0,216 & $1,81(0,65-5,03)$ & 0,252 \\
\hline 4 & $3,88(1,55-9,71)$ & 0,004 & $4,33(1,68-11,11)$ & 0,002 \\
\hline & \multicolumn{4}{|c|}{ Incentivo à diálise } \\
\hline
\end{tabular}

Nível de Ativação

\begin{tabular}{ccccc}
$\mathbf{1}$ & 1.0 & \multicolumn{1}{c}{1.0} & \\
$\mathbf{2}$ & $4,70(1,73-12,75)$ & $\mathbf{0 , 0 0 2}$ & $5,20(1,86-14,53)$ & $\mathbf{0 , 0 0 2}$ \\
$\mathbf{3}$ & $0,65(0,24-1,78)$ & 0,411 & $0,70(0,25-1,90)$ & 0,501 \\
$\mathbf{4}$ & $2,10(0,85-5,18)$ & 0,107 & $2,16(0,86-5,14)$ & 0,100 \\
\hline
\end{tabular}

† Representação da amostra de acordo com os componentes de qualidade de vida com pontuação acima do percentil 50. Domínio Sintoma $\geq 81,25$, n=84;

Funcionamento físico $\geq 60, n=79$; Saúde geral $\geq 65, n=79$; Bem-estar emocional $\geq 75, n=79$; Energia/Fadiga $\geq 65, n=81 ;$ Componente mental $\geq 47,95, n=81$.

* Ajuste 1: Sexo e Idade. ** Ajuste 2: Sexo; Idade; Tempo de Hemodiálise; Número de Comorbidades; Hemoglobina e KTV. Fonte: dados de pesquisa. 
além da presença de dor e ansiedade, quando comparados aos pacientes com alta ativação (níveis 3 e 4).4,25,26

Neste estudo, pacientes com nível 4 de ativação possuíam mais chances de ter melhor QVRS no composto saúde mental em relação aos pacientes do nível 1 de ativação. Em pesquisa anterior, que envolveu pacientes em todos os níveis da DRC, uma pior ativação esteve associada à pior pontuação no componente de saúde mental nos homens. ${ }^{16}$ Ao considerar a alta prevalência de ansiedade e depressão nessa população, ${ }^{27,28}$ o auxílio para o aumento da ativação pode ser uma estratégia eficaz para reduzir os transtornos mentais e, consequentemente, melhorar a saúde mental e a QVRS desses indivíduos.

Neste estudo, pacientes que apresentaram nível de ativação mais alto possuíam mais chances de ter melhor QVRS, considerando o domínio sintoma. Diferentemente do achado deste estudo, em pesquisa realizada com 305 pessoas em todos os estágios da DRC, a ativação não se associou a esse domínio. ${ }^{16} \mathrm{E}$ uma possível justificativa para essa divergência pode ser a diferença entre os estágios da DRC em que se encontravam os participantes que compunham as duas amostras dos estudos, uma vez que a presença de sintomas varia conforme a gravidade da DRC.

No KDOL-SF, o sintoma é considerado um domínio para mensurar a QVRS, contudo, existem instrumentos que avaliam a presença de sintomas relacionados à DRC. Nesse sentido, o estudo da Associação Renal do Reino Unido $(2020)^{4}$ analisou a relação entre a carga de sintomas, por meio de 17 itens de mensuração de sintoma do instrumento POS-S, e a ativação de pacientes renais crônicos. Observou-se que quanto maior a ativação, menor a carga de sintomas. Ao considerar cada um dos 17 itens do instrumento individualmente e a carga geral deles, também se reforça o achado deste estudo de que uma maior ativação está relacionada com uma melhor QVRS no domínio sintoma. Esta relação pode ser justificada pelo fato de que pessoas mais ativadas apresentam melhores indicadores clínicos como, por exemplo, níveis séricos de eletrólitos adequados. ${ }^{29}$ Esses eletrólitos, quando aumentados nos pacientes em hemodiálise (por exemplo, potássio, fósforo e sódio), ocasionam sintomas como câimbras, náuseas, diarreia e astenia.

Vale ressaltar que, dentre os 17 sintomas avaliados pela Associação Renal do Reino Unido (2020) e associados com a ativação do paciente, o sintoma fraqueza ou falta de energia foi o mais prevalente entre os entrevistados e a significância desta relação corrobora o achado deste estudo. Os pacientes com nível 4 de ativação possuíam mais chances de melhor QVRS, considerando o domínio energia/fadiga, quando comparados aos pacientes com nível 1 de ativação. ${ }^{4}$

No que se refere à saúde geral, pessoas com nível 4 de ativação possuíam mais chances de ter melhor pontuação em relação às pessoas do nível 1. Estudos anteriores envolvendo pacientes em HD, ${ }^{16}$ com doença pulmonar obstrutiva crônica, insuficiência cardíaca congestiva, DRC (TFG<60 ml/min/1,73m²) e Diabetes Mellitus tipo II,, ${ }^{30,31}$ também reiteram que quanto maior a ativação, melhor será a saúde autorreferida. Uma possível explicação para essa relação é que pessoas com maiores níveis de ativação possuem melhores desfechos clínicos e apresentam condutas mais saudáveis do que pessoas pouco ativadas, ${ }^{22,29,32}$ e estas variáveis interferem na saúde geral do indivíduo.

Possuir o nível 4 de ativação aumentou as chances de os pacientes em HD apresentarem melhor pontuação no domínio funcionamento físico da QVRS. Ao considerar que a mobilidade é influenciada pelo funcionamento físico, como descrito anteriormente, há evidências entre a associação da mobilidade com a ativação de pacientes com DRC, ${ }^{4}$ o que corrobora o achado deste estudo.

Com relação às associações entre o bem-estar emocional e a ativação, não se encontraram outros estudos que explorassem essa associação em pacientes com DRC. Mas, em uma pesquisa que envolveu pacientes com obesidade grave e que realizaram a cirurgia bariátrica, a ativação e o bem-estar emocional foram positivamente associados $(B=0,48, p<0,001)$. Vale ressaltar que os autores mensuraram o bem-estar emocional por meio de um instrumento específico e não por meio de um domínio da QVRS. ${ }^{33}$

Em estudo anterior, que mensurou os fatores associados à ativação de pacientes com diabetes comórbidos e DRC, uma pior carga da doença renal esteve associada à pior QVRS de pacientes do grupo de baixa ativação (níveis 1 e 2) quando comparados com os de alta ativação (níveis 3 e 4), ${ }^{16}$ diferentemente dos achados deste estudo nos quais não houve associação entre o domínio carga da DRC e o nível de ativação. Essa divergência pode ser justificada pelo fato de este estudo restringir-se a pacientes em HD, enquanto, no outro, foram incluídas 305 pessoas com DRC nos estágios 3 a 5, sendo que, destas, apenas 59 estavam em diálise. ${ }^{16}$ Nesse sentido, há evidências de que a ansiedade, a inquietação e o medo são mais presentes nos estágios iniciais da DRC, ${ }^{34}$ o que pode influenciar esse domínio da QVRS.

Diante do exposto, evidencia-se a relação positiva entre a ativação do paciente e a QVRS. Ao considerar que as intervenções na ativação são capazes de melhorar a qualidade de vida, ${ }^{35,36}$ os profissionais de saúde devem estabelecer intervenções que considerem o nível de ativação dos pacientes dialíticos, visando a fortalecer o autogerenciamento da saúde neles e, assim, obter melhores resultados de saúde como a QVRS. ${ }^{35}$

\section{CONCLUSÃO E IMPLICAÇÕES PARA A PRÁTICA}

Este estudo relacionou o nível de ativação com a QVRS de pacientes em hemodiálise e pessoas altamente ativadas (nível 4 de ativação) apresentaram maiores chances de melhor QVRS nos domínios sintomas, funcionamento físico, saúde geral, bemestar emocional, energia/fadiga e componente mental.

Como foi realizado um estudo transversal, não se pode descartar a causalidade reversa, ou seja, que uma maior QVRS pode influenciar o nível de ativação do paciente, tornando-se uma limitação desta investigação. Nesse sentido, visando a elucidar essa relação causal, sugere-se a realização de estudos longitudinais. Além dessa, outra limitação foi o tipo de amostragem por conveniência, o que impossibilita fazer inferências para outras populações de pacientes em hemodiálise. 
Como implicações para a prática, salienta-se que o nível de ativação se associa à QVRS de pacientes em tratamento hemodialítico. Assim, os profissionais de saúde podem utilizar essa medida ao implementar estratégias que visem ao aumento da QVRS dessa população.

\section{AGRADECIMENTOS}

Agradece-se à Universidade Federal de Juiz de Fora pela concessão da bolsa do Programa de Monitoria de Pós-Graduação à Denise Rocha Raimundo Leone.

\section{CONTRIBUIÇÕES DOS AUTORES}

Desenho do estudo. Aquisição, análise de dados e interpretação dos resultados. Redação e revisão crítica do manuscrito. Aprovação da versão final do artigo. Responsabilidade por todos os aspectos do conteúdo e pela integridade do artigo publicado. Denise Rocha Raimundo Leone

Análise de dados e interpretação dos resultados. Redação e revisão crítica do manuscrito. Aprovação da versão final do artigo. Responsabilidade por todos os aspectos do conteúdo e pela integridade do artigo publicado. Gabriela Amorim Pereira

Análise de dados e interpretação dos resultados. Redação e revisão crítica do manuscrito. Aprovação da versão final do artigo. Responsabilidade por todos os aspectos do conteúdo e pela integridade do artigo publicado. Arison Cristian de Paula Silva

Desenho do estudo. Análise de dados e interpretação dos resultados. Redação e revisão crítica do manuscrito. Aprovação da versão final do artigo. Responsabilidade por todos os aspectos do conteúdo e pela integridade do artigo publicado. Aline Silva de Aguiar

\section{EDITOR ASSOCIADO}

\section{Cândida Caniçali Primo}

\section{REFERÊNCIAS}

1. Neves PDMM, Sesso RCC, Thomé FS, Lugon JR, Nasicmento MM Brazilian Dialysis Census: analysis of data from the 2009-2018 decade. J Bras Nefrol. 2020;42(2):191-200. http://dx.doi.org/10.1590/21758239-jbn-2019-0234. PMid:32459279.

2. Pecoits-Filho R, Rosa-Diez G, Gonzalez-Bedat M, Marinovich S, Fernandez S, Lugon $\mathrm{J}$ et al. Renal replacement therapy in CKD: an update from the Latin American Registry of Dialysis and Transplantation. J. Braz. Nephrol. 2015;37(1):9-13. http://dx.doi.org/10.5935/01012800.20150002. PMid:25923744.

3. United States Renal Data System. USRDS Annual Data Report: epidemiology of kidney disease in the United States. National Institutes of Health, National Institute of Diabetes and Digestive and Kidney Diseases. Bethesda (MD): United States Renal Data System; 2019

4. The Renal Association. Transforming participation in chronic kidney disease [Internet]. Bristol: The Renal Association; 2019 [citado 2020 Ago 6]. Disponível em: https://www.thinkkidneys.nhs.uk/ckd/wp-content uploads/sites/4/2019/01/Transforming-Participation-in-Chronic-KidneyDisease-1.pdf

5. Macêdo IS, Macêdo GS, Pacheco ES, Mota MS, de Sousa ARR Support and coping strategies used by chronic renal patients undergoing hemodialysis. RSD. 2020;9(9):e340996908. http://dx.doi.org/10.33448/ rsd-v9i9.6908.

6. Zazzeroni L, Pasquinelli G, Nanni E, Cremonini V, Rubbi I. Comparison of quality of life in patients undergoing hemodialysis and peritoneal dialysis: a systematic review and meta-analysis. Kidney Blood Press Res. 2017;42(4):717-27. http://dx.doi.org/10.1159/000484115. PMid:29049991.

7. Wang R, Tang C, Chen X, Zhu C, Feng W, Li P et al. Poor sleep and reduced quality of life were associated with symptom distress in patients receiving maintenance hemodialysis. Health Qual Life Outcomes. 2016;14(125):125. http://dx.doi.org/10.1186/s12955-016-0531-6. PMid:27608683.

8. Centers for Disease Control and Prevention. HRQOL concepts [Internet] 2020 [citado 2020 Out 25]. Disponível em: http://www.cdc.gov/hrqol/ concept.htm

9. Oliveira APB, Schmidt DB, Amatneeks TM, Santos JC, Cavallet LH, Michel RB. Quality of life in hemodialysis patients and the relationship with mortality, hospitalizations and poor treatment adherence. J Bras Nefrol. 2016;38(4):411-20. http://dx.doi.org/10.5935/0101-2800.20160066. PMid:28001183.

10. Wright LS, Wilson L. Quality of life and self-efficacy in three dialysis modalities: Incenter hemodialysis, home hemodialysis, and home peritonea dialysis. J Bras Nefrol. 2015;42(5):463-76, quiz 477. PMid:26591271.

11. Gonçalves FA, Dalosso IF, Borba JMC, Bucaneve J, Valerio NMP Okamoto CT et al. Quality of life in chronic renal patients on hemodialysis or peritoneal dialysis: a comparative study in a referral service of Curitiba - PR. Braz. J. Nephrol. 2015;37(4):467-74. http://dx.doi.org/10.5935/01012800.20150074. PMid:26648496.

12. Blakemore A, Hann M, Howells K, Panagioti M, Sidaway M, Reeves $D$ et al. Patient activation in older people with long-term conditions and multimorbidity: correlates and change in a cohort study in the United Kingdom. BMC Health Serv Res. 2016;16:582. http://dx.doi.org/10.1186/ s12913-016-1843-2.

13. Hibbard JH, Stockard J, Mahoney ER, Tusler M. Development of the Patient Activation Measure (PAM): conceptualizing and measuring activation in patients and consumers. Health Serv Res. 2004;39(4 Pt 1):1005-26. http://dx.doi.org/10.1111/j.1475-6773.2004.00269.x. PMid:15230939.

14. Newland P, Lorenz R, Oliver BJ. Patient activation in adults with chronic conditions: a systematic review. J Health Psychol. 2021 jan;26(1):103 14. http://dx.doi.org/10.1177/1359105320947790. PMid:32830587.

15. Sánchez-Cabezas AM, Morillo-Gallego N, Merino-Martínez RM, Crespo-Montero R. Calidad de vida de los pacientes en diálisis. Revisión sistemática. Enferm Nefrol. 2019;22(3). http://dx.doi.org/10.4321/ s2254-28842019000300003.

16. Zimbudzi E, Lo C, Ranasinha S, Fulcher GR, Jan S, Kerr PG et al Factors associated with patient activation in an Australian population with comorbid diabetes and chronic kidney disease: a cross sectional study. BMJ Open. 2017;7(10):e017695. http://dx.doi.org/10.1136/ bmjopen-2017-017695. PMid:29061622.

17. Van Bulck L, Claes K, Dierickx K, Hellemans A, Jamar S, Smets S et al. Patient and treatment characteristics associated with patient activation in patients undergoing hemodialysis: a cross-sectional study. BMC Nephrol. 2018;19(126):126. http://dx.doi.org/10.1186/s12882-0180917-2. PMid:29859047.

18. Associação Brasileira de Empresas de Pesquisa. Critério de Classificação econômica Brasil-2018 [Internet]. São Paulo: ABEP; 2018 [citado 2020 Ago 8]. Disponível em: http://www.abep.org/criterio-brasil

19. Hays RD, Joel K, Donna M, Stephen C, Naseen A, William BC et al. Kidney Disease Quality of Life Short Form (KDQOL-SF TM), version 1.3: a manual for use and scoring [Internet]. Santa Monica, CA: RAND Corporation; 1997 [citado 2020 Ago 25]. Disponível em: https://www. rand.org/pubs/papers/P7994.html

20. Duarte PS, Miyazaki MCOS, Ciconelli RM, Sesso R. Tradução e adaptação cultural do instrumento de avaliação de qualidade de vida para pacientes renais crônicos (KDQOL-SFTM). Rev Assoc Med Bras. 2003;49(4):375-81. http://dx.doi.org/10.1590/S0104-42302003000400027. PMid:14963588. 
21. Cunha CM, da Cunha DCPT, Manzato RO, Nepomuceno E, da Silva D, Dantas RAS. Validation of the Brazilian Version of the Patient Activation Measure 13. J Nurs Meas. 2019;27(1):97-113. https://doi. org/10.1891/1061-3749.27.1.97. PMID: 31068494.

22. Greene J, Hibbard JH, Sacks R, Overton V, Parrotta CD. When patient activation levels change, health outcomes and costs change, too. Health Aff (Millwood). 2015;34(3):431-7. http://dx.doi.org/10.1377/ hlthaff.2014.0452. PMid:25732493.

23. Insignia Health. What the Patient Activation Measure ${ }^{\circledR}$ Reveals. USA: Insignia Health; 2017. TM Licence Materials.

24. Santos PR, Pontes LRSK. Mudança do nível de qualidade de vida em portadores de insuficiência renal crônica terminal durante seguimento de 12 meses. Rev Assoc Med Bras. 2007 ago;53(4):329-34. http:// dx.doi.org/10.1590/S0104-42302007000400018. PMid:17823736.

25. Herdman M, Gudex C, Lloyd A, Janssen MF, Kind P, Parkin D et al. Development and preliminary testing of the new five-level version of EQ-5D (EQ-5D-5L). Qual Life Res. 2011;20(10):1727-36. http://dx.doi. org/10.1007/s11136-011-9903-x. PMid:21479777.

26. Brooks R. EuroQol: the current state of play. Health Policy. 1996;37(1):5372. http://dx.doi.org/10.1016/0168-8510(96)00822-6. PMid:10158943.

27. Sousa L, Valentim O, Marques-Vieira C, Antunes AV, Severino S, José $\mathrm{H}$. Association between stress/anxiety, depression, pain and quality of life in people with chronic kidney disease. Rev Port Enferm Saude Mental. 2020;23. http://dx.doi.org/10.19131/rpesm.0272.

28. Schmidt DB. Quality of life and mental health in hemodialysis patients: a challenge for multiprofessional practices. J Bras Nefrol. 2019;41(1):10-1. http://dx. doi.org/10.1590/2175-8239-jbn-2018-0227. PMid:31063179.

29. Hibbard JH, Mahoney ER, Sonet E. Does patient activation level affect the cancer patient journey? Patient Educ Couns. 2017;100(7):1276-9. http://dx.doi.org/10.1016/j.pec.2017.03.019. PMid:28330715.
30. Bos-Touwen I, Schuurmans M, Monninkhof EM, Korpershoek Y, Spruit-Bentvelzen L, Ertugrul-van der Graaf I et al. Patient and disease characteristics associated with activation for self-management in patients with diabetes, chronic obstructive pulmonary disease, chronic heart failure and chronic renal disease: a cross-sectional survey study PLoS One. 2015;10(5):e0126400. http://dx.doi.org/10.1371/journal. pone.0126400. PMid:25950517.

31. Hendriks M, Rademakers J. Relationships between patient activation, disease-specific knowledge and health outcomes among people with diabetes; a survey study. BMC Health Serv Res. 2014;14(393):393. http://dx.doi.org/10.1186/1472-6963-14-393. PMid:25227734.

32. Greene J, Hibbard JH, Sacks R, Overton V, Parrotta CD. When patient activation levels change, health outcomes and costs change, too. Health Aff (Millwood). 2015;34(3):431-7. http://dx.doi.org/10.1377/ hlthaff.2014.0452. PMid:25732493.

33. Solberg H, Aarflot M, Skotnes LH, Graue M. Relationships between emotional well-being, patient activation and social support among adults with severe obesity who have undergone gastric bypass surger. Open J Nurs. 2015;5(7):596-604. http://dx.doi.org/10.4236/ojn.2015.57063.

34. Johnson ML, Zimmerman L, Welch J, Hertzog M, Pozehl B, Plumb T. Patient activation with knowledge, self-management and confidence in chronic kidney disease. J Ren Care. 2016;42(1):15-22. http://dx.doi. org/10.1111/jorc.12142. PMid:26537188.

35. Lin MY, Weng WS, Apriliyasari RW, Van Truong P, Tsai PS. Effects of patient activation intervention on chronic diseases: a meta-analysis. J Nurs Res. 2020;28(5):e116. http://dx.doi.org/10.1097/jnr.0000000000000387. PMid:32649394.

36. Nelson RG, Pankratz V, Ghahate D, Bobelu J, Faber T, Shah V. Homebased kidney care, patient activation, and risk factors for CKD progression in Zuni Indians: a randomized, controlled clinical trial. Clin J Am Soc Nephrol.2018;13(12):1801-9. http://dx.doi.org/10.2215/CJN.06910618. PMid:30442864. 